\title{
Neutrino-Nucleus Scattering Brief
}

\author{
Michael Wagman
}

\section{Snowmass 2021 \\ Community Planning Meeting}

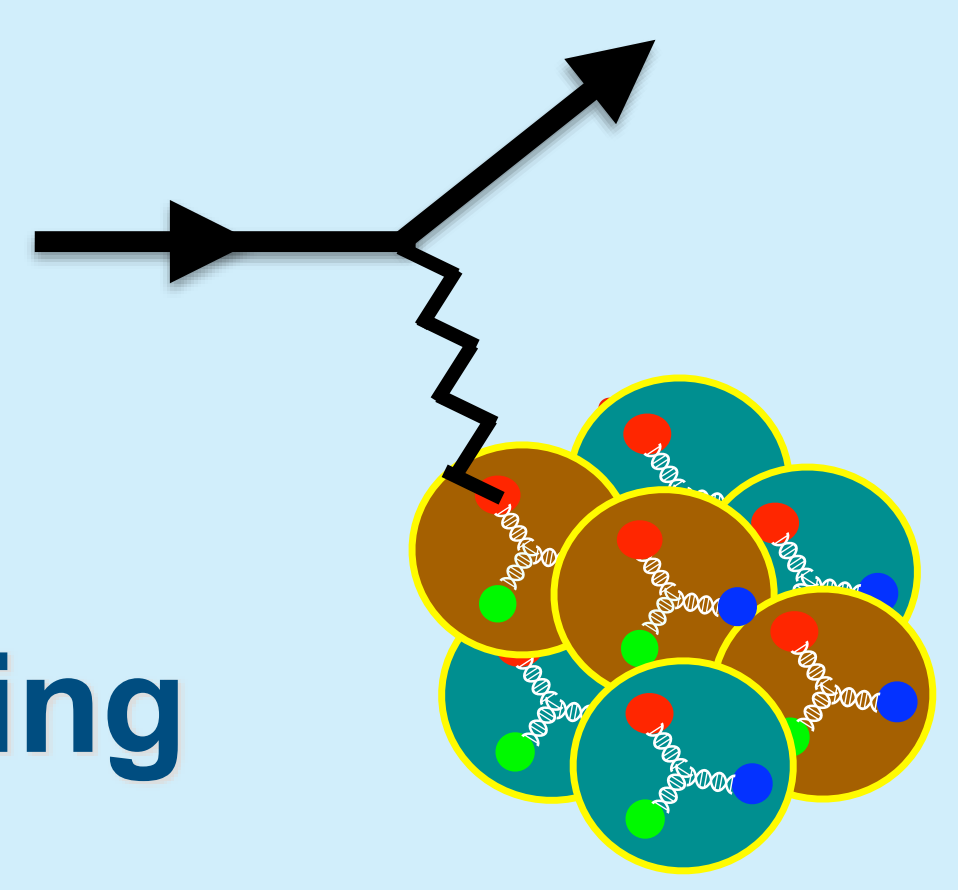

Oct 5, 2020

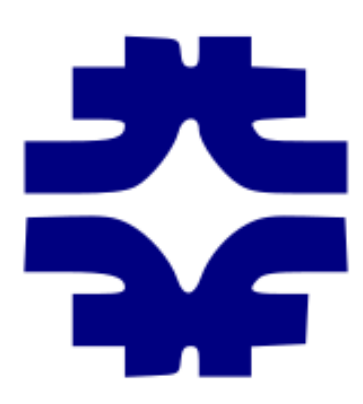




\section{The precision era of $\nu$ physics}

Next-generation oscillation experiments can answer fundamental questions about the neutrino mass ordering, size of leptonic $C P$ violation, and more

Discovering new physics with $\nu$ experiments requires control of all the Standard Model physics in the detector

Acciarri et al (DUNE) arXiv 1512.06148

$$
\underbrace{N_{\mathrm{FD}}^{\text {expected }}\left(\nu_{e}\right)}_{\text {Measured }}=\underbrace{N_{\mathrm{ND}}^{\text {data }}\left(\nu_{\mu}\right)}_{\nu A \text { cross-section }} \otimes \underbrace{\Phi_{\mathrm{FD}}\left(\nu_{\mu}\right)}_{\text {Goal }} \otimes \underbrace{P\left(\nu_{\mu} \rightarrow \nu_{e}\right)}_{\Phi_{\mathrm{ND}}\left(\nu_{\mu}\right)} \otimes \underbrace{\sigma_{\sigma_{\mathrm{ND}}\left(\nu_{\mu}\right)}^{\sigma_{\mathrm{FD}}\left(\nu_{e}\right)}}_{\frac{\varepsilon_{\mathrm{FD}}\left(\nu_{e}\right)}{\varepsilon_{\mathrm{ND}}\left(\nu_{\mu}\right)}}
$$

Exclusive $\nu A$ cross-sections required for event-by-event reconstruction of incident neutrino energy
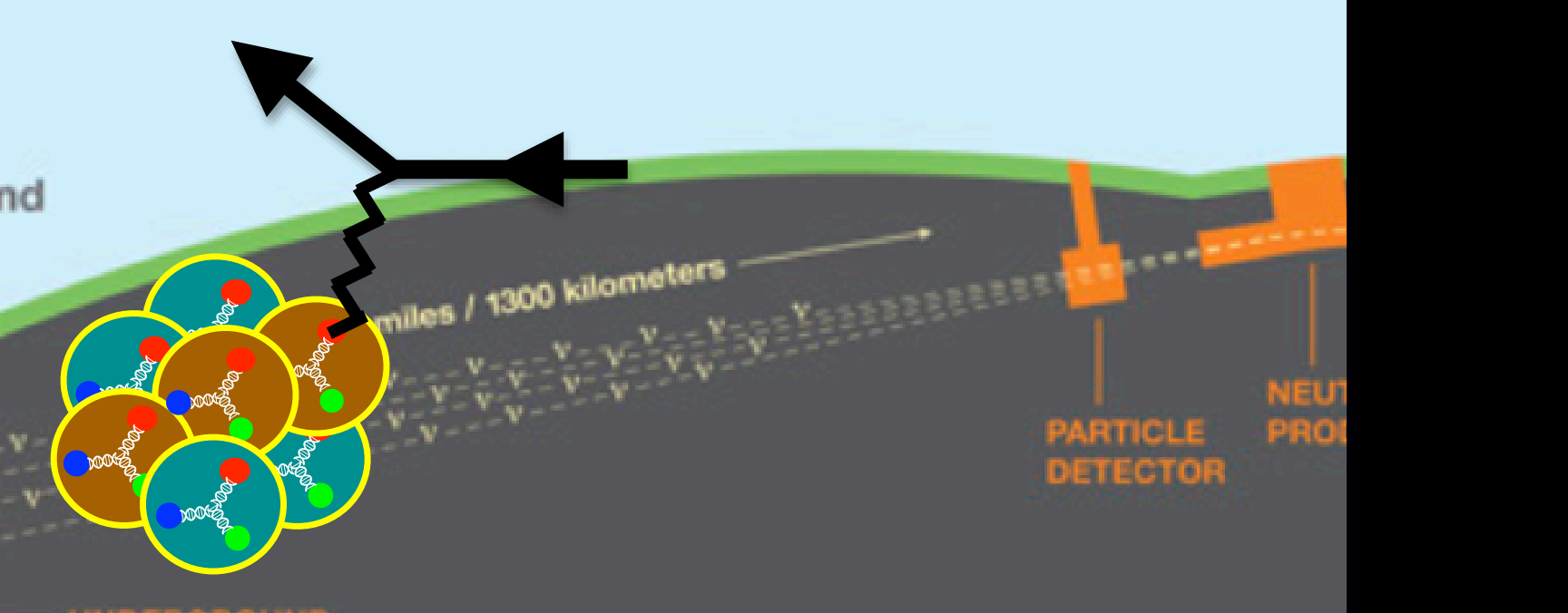


\section{Challenges of $\nu A$}

Neutrino-argon cross-sections with few percent-level accuracy required to achieve design sensitivity to $C P$ violation at DUNE

Acciarri et al (DUNE) arXiv 1512.06148
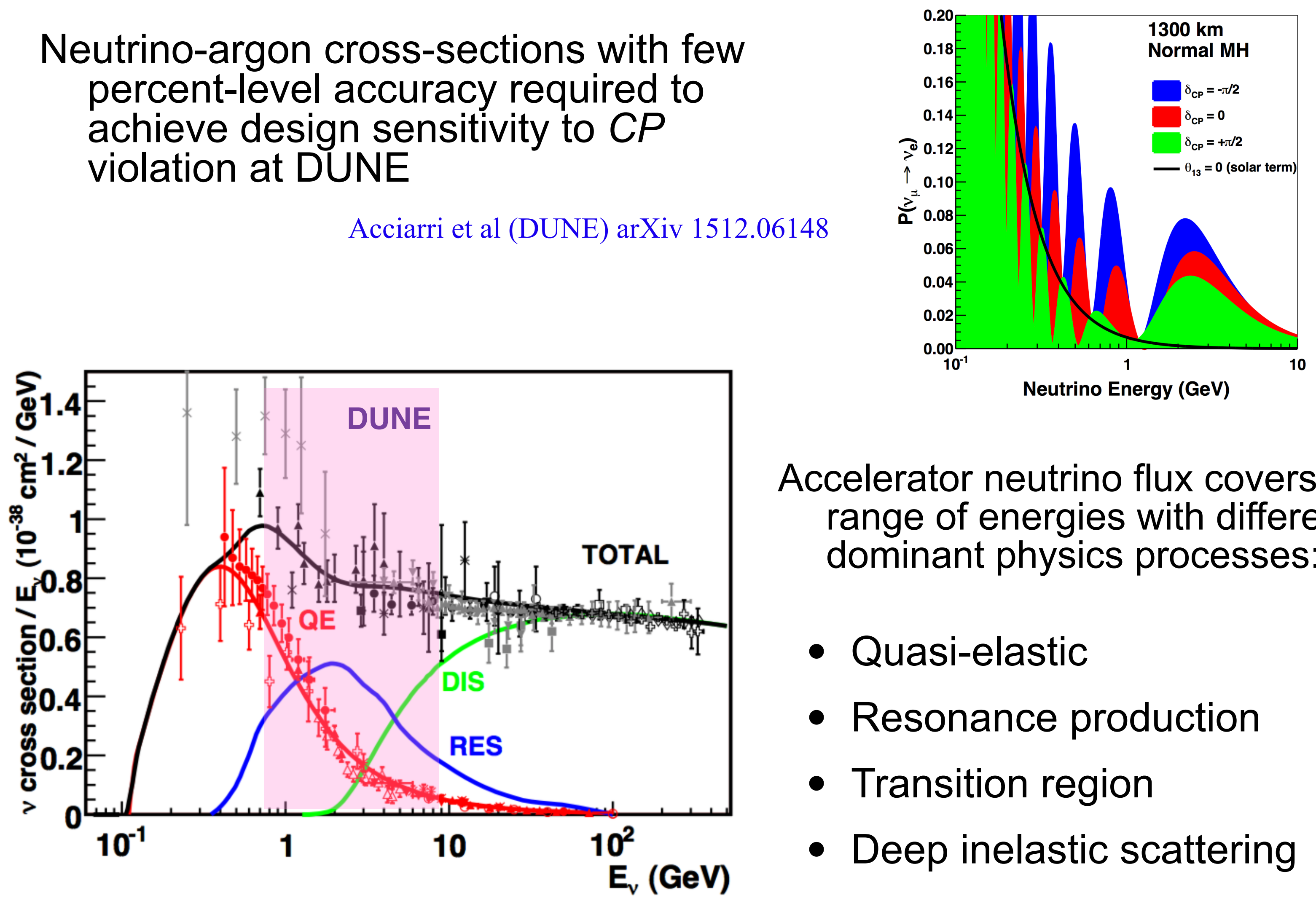

Accelerator neutrino flux covers a wide range of energies with different dominant physics processes:

- Quasi-elastic

- Resonance production

- Transition region

- Deep inelastic scattering 
Event Generators for Accelerator-Based Neutrino Experiments

Contact Information:

Joshua Isaacson (Theoretical Physics Department, Fermilab) [isaacson@fnal.gov]

William I. Jay (Theoretical Physics Department, Fermilab) [wjay@fnal.gov]

Alessandro Lovato (INFN-TIFPA, Trento, Italy; Physics Division, Argonne National Lab) [lovato@anl.gov]

Pedro A. N. Machado (Theoretical Physics Department, Fermilab) [pmachado@fnal.gov]

Noemi Rocco (Theoretical Physics Department, Fermilab; Physics Division, Argonne National Lab)

[nrocco@anl.gov]

Incorporating all constraints on $\nu A$ from experiment and LQCD in neutrino Monte Carlo event generators is critical

Factorization of leptonic and hadronic parts allows easier verification of nuclear models (e.g. vs electron scattering, where generator progress is still needed) and searches for novel new physics signatures

A coordinated framework of modular event generator components would simplify comparing and understanding systematic uncertainties of generators

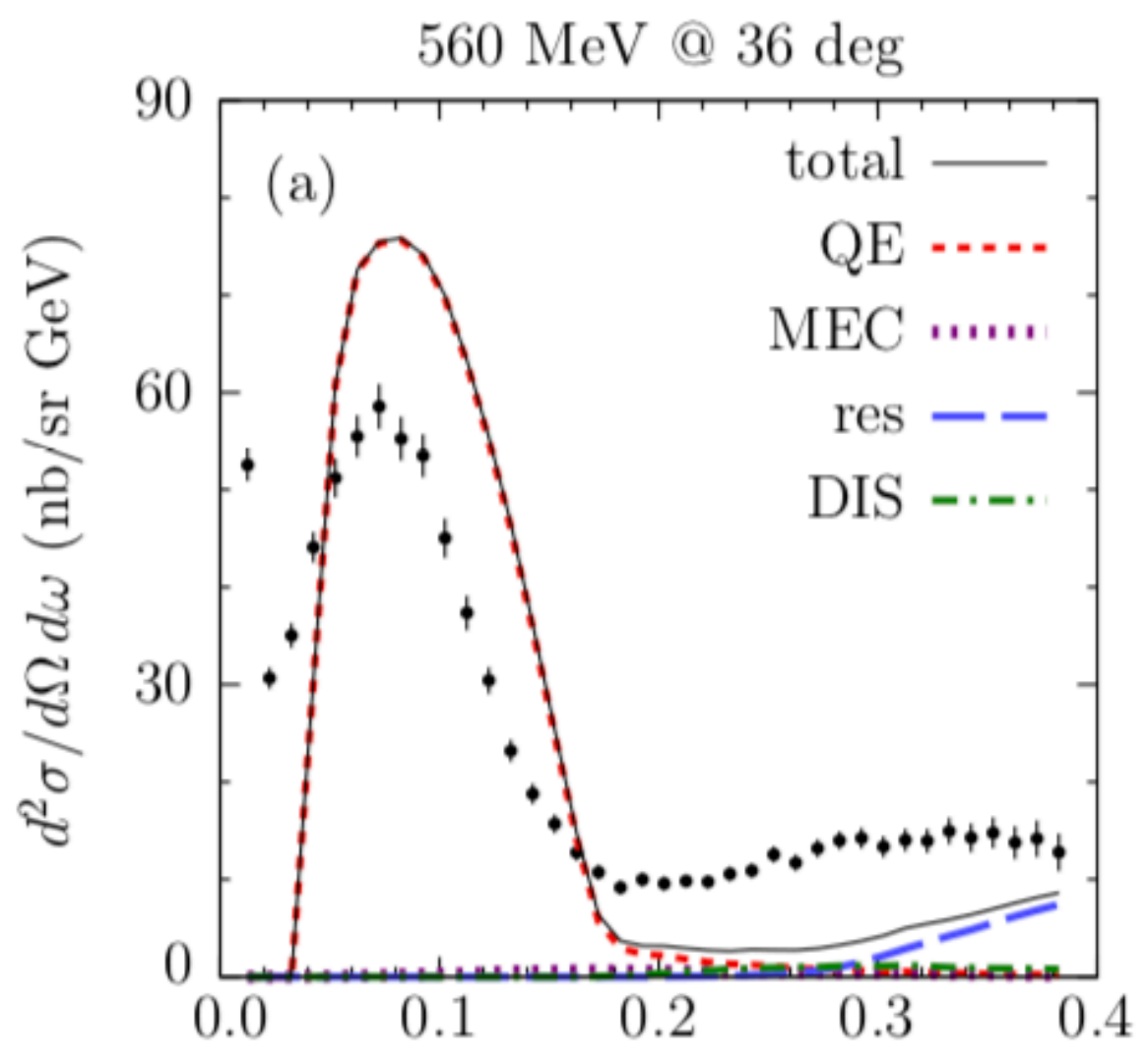

Ankowski, Friedland PRD 102 (2020) 


\section{Lattice QCD and $\nu A$}

LQCD can provide accurate constraints on $\nu A$ scattering across energies

See USQCD $\nu A$ white paper: Kronfeld et al Eur. Phys. J. A 55 (2019)
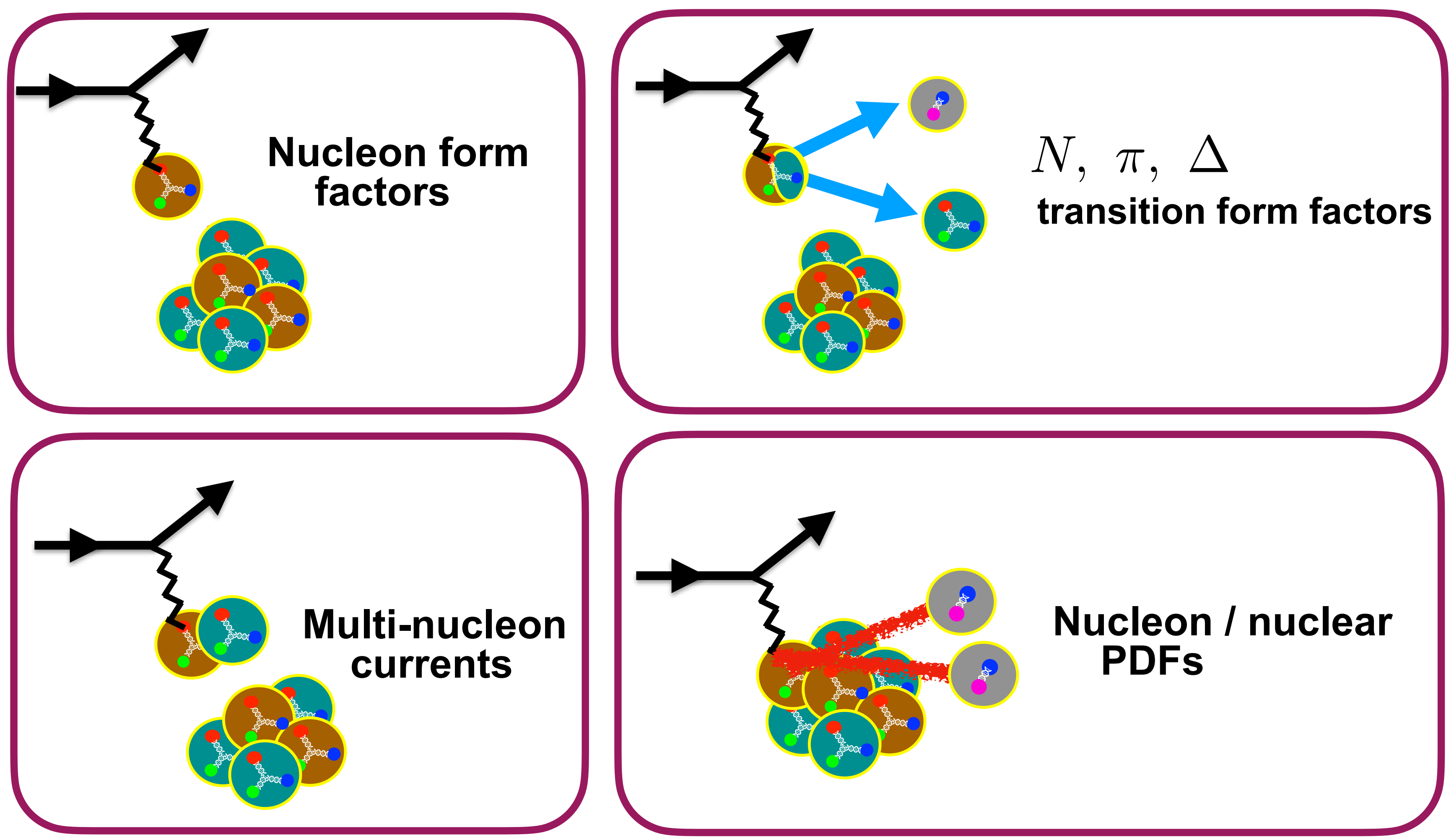


\section{Lattice QCD and $\nu A$}

\section{Snowmass 2021 LoI: Nucleon Form Factors for Neutrino Physics}

Taku Izubuchi, ${ }^{\mathrm{a}, \mathrm{b}}$ Christoph Lehner, ${ }^{\mathrm{a}, \mathrm{c}}$ Aaron S. Meyer, ${ }^{a *}$ Shigemi Ohta ${ }^{\mathrm{b}, \mathrm{d}, \mathrm{e}}$, Sergey Syritsyn ${ }^{\mathrm{b}, \mathrm{f}}$

Lattice-QCD Calculations Supporting Neutrino-Oscillation

Experiments

Fermilab Lattice and MILC Collaborations

\section{Theoretical predictions of Neutrino-nucleus Interactions}

\section{Contact Information:}

Rajan Gupta (Los Alamos National Laboratory) [rajan@lanl.gov]:

Stefano Gandolfi (Los Alamos National Laboratory) [stefano@lanl.gov]:

Lattice Calculation of Neutrino-Nucleon Cross Section

Keh-Fei Liu ${ }^{1}$ (liu@g.uky.edu), Terrence Draper ${ }^{1}$, Jian Liang ${ }^{1}$, G. Wang ${ }^{1}$, Yi-Bo Yang ${ }^{2}$, and Yong Zhao

\section{Connecting QCD to neutrino-nucleus scattering}

Joseph Carlson ${ }^{1}$, Chia Cheng Chang (張家丞 $)^{2,3,4}$, William Detmold ${ }^{5}$, Joshua Isaacson ${ }^{6}$, William Jay ${ }^{6}$, Gurtej Kanwar ${ }^{5}$, Andreas Kronfeld ${ }^{6}$, Huey-Wen Lin ${ }^{7}$, Yin Lin (林/䏍 $)^{6,8}$,

Keh-Fei Liu ${ }^{9}$, Alessandro Lovato ${ }^{10,11}$, Pedro Machado ${ }^{6}$, Aaron S. Meyer ${ }^{12}$, Saori

Pastore $^{13}$, Noemi Rocco ${ }^{6,10}$, Phiala Shanahan ${ }^{5}$, and Michael Wagman ${ }^{6}$ 


\section{Nucleon form factors}

$\nu A$ scattering amplitudes factorize into leptonic and hadronic parts

$$
\mathcal{M}_{\nu A \rightarrow \ell f} \propto\left[\bar{u}_{\ell} \gamma_{\mu}\left(\frac{1-\gamma_{5}}{2}\right) u_{\nu}\right]\left\langle f\left|\bar{q} \gamma_{\mu}\left(\frac{1-\gamma_{5}}{2}\right) q\right| A\right\rangle
$$

Single-nucleon currents dominate at low energies where nucleons are effective degrees of freedom for nuclei, multi-nucleon currents provide corrections
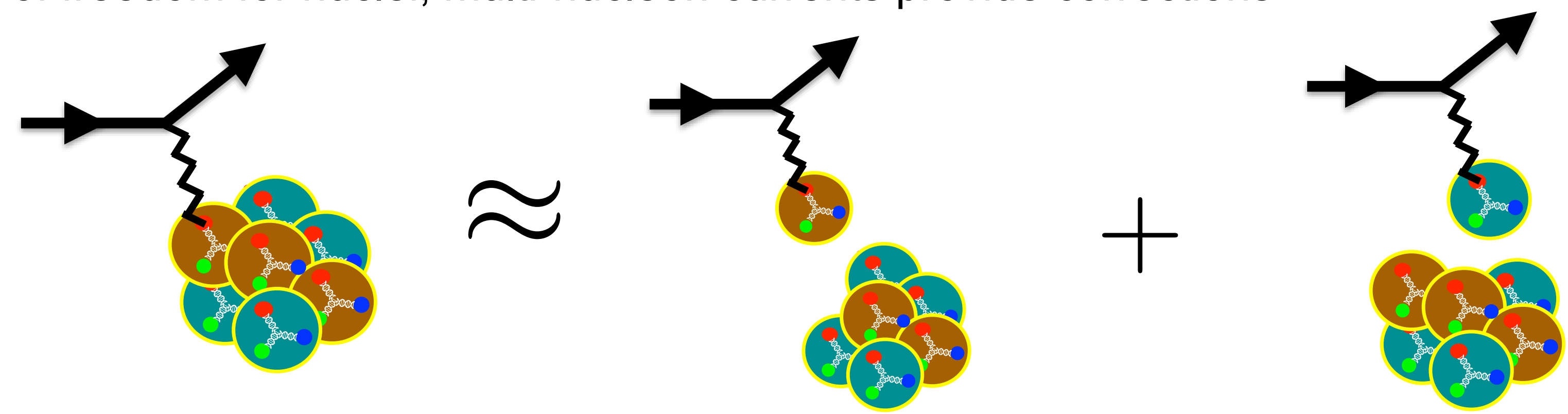

Nucleon vector and axial form factors are key inputs to nuclear EFTs / models

$$
\left\langle N\left(p^{\prime}\right)\left|A_{\mu}(q)\right| N(p)\right\rangle=\bar{u}\left(p^{\prime}\right)\left[G_{A}\left(Q^{2}\right) \gamma_{\mu} \gamma_{5}+\frac{\widetilde{G}_{P}\left(Q^{2}\right)}{2 M_{N}} q_{\mu} \gamma_{5}\right] u(p)
$$

Vector form factors measured in electron scattering, axial form factors less constrained 


\section{Nucleon form factors}

Calculations of vector current form factors performed at physical quark masses:
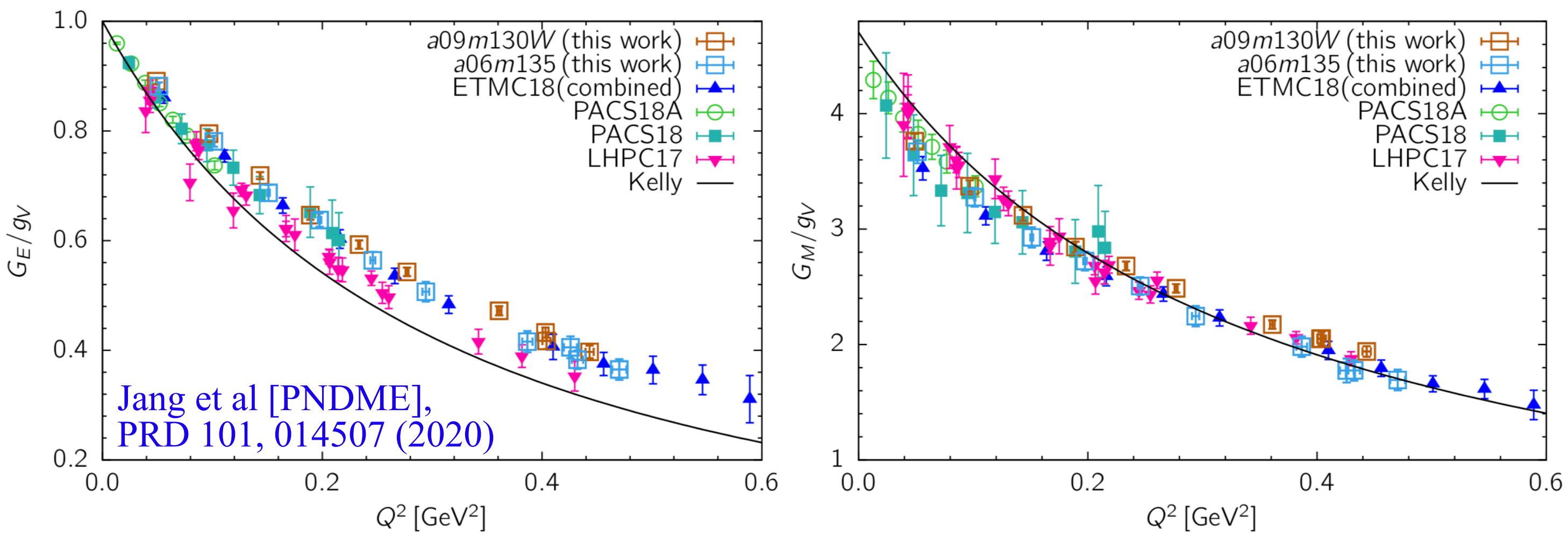

Recent axial form factors studies demonstrated the importance of $N \pi$ excited-state effects in achieving controlled systematic uncertainties
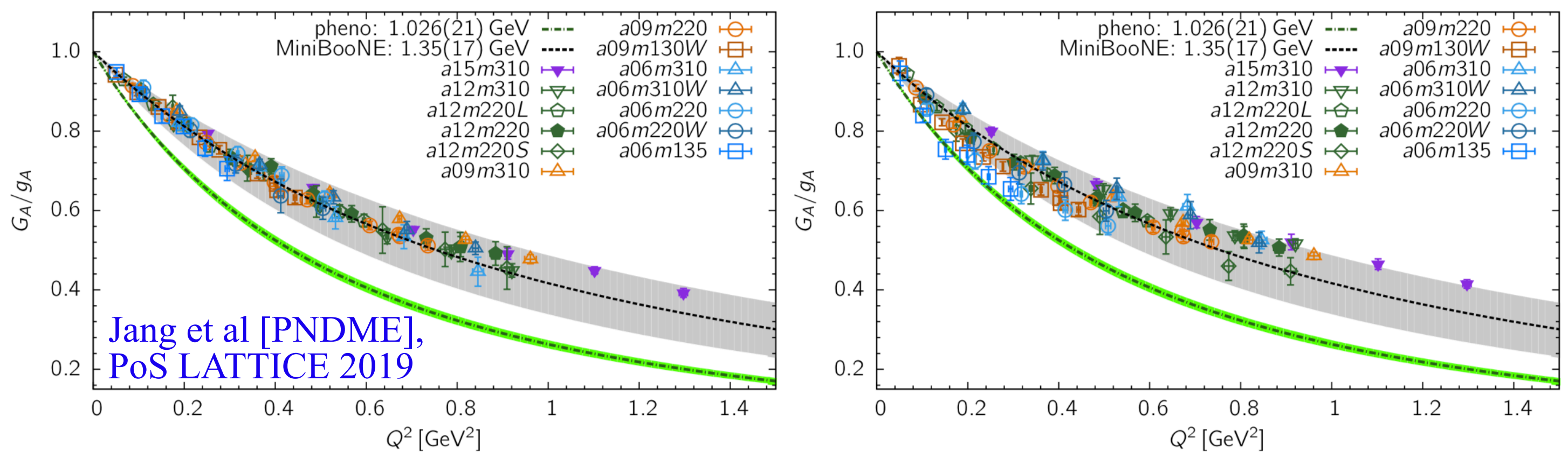


\section{Nucleon form factors}

Neutrino Scattering Measurements on Hydrogen and Deuterium

\section{Contact Information:}

Richard Hill (University of Kentucky and Fermilab) richard.hill@uky.edu Tom Junk (Fermilab) trj@fnal.gov

Nucleon form factors also directly accessible without nuclear uncertainties in experiments using elementary targets

Neutrino scattering with hydrogen and deuterium provides clean environment for measuring nucleon form factors and simplest nuclear effects

Options include solid $\mathrm{H}$ target (straw tube tracker), liquid- $\mathrm{H}_{2} / \mathrm{D}_{2}$ bubble chamber, and high-pressure gas TPC ( $\mathrm{H}$ mixture, $\mathrm{He}, \ldots)$ at DUNE near detector

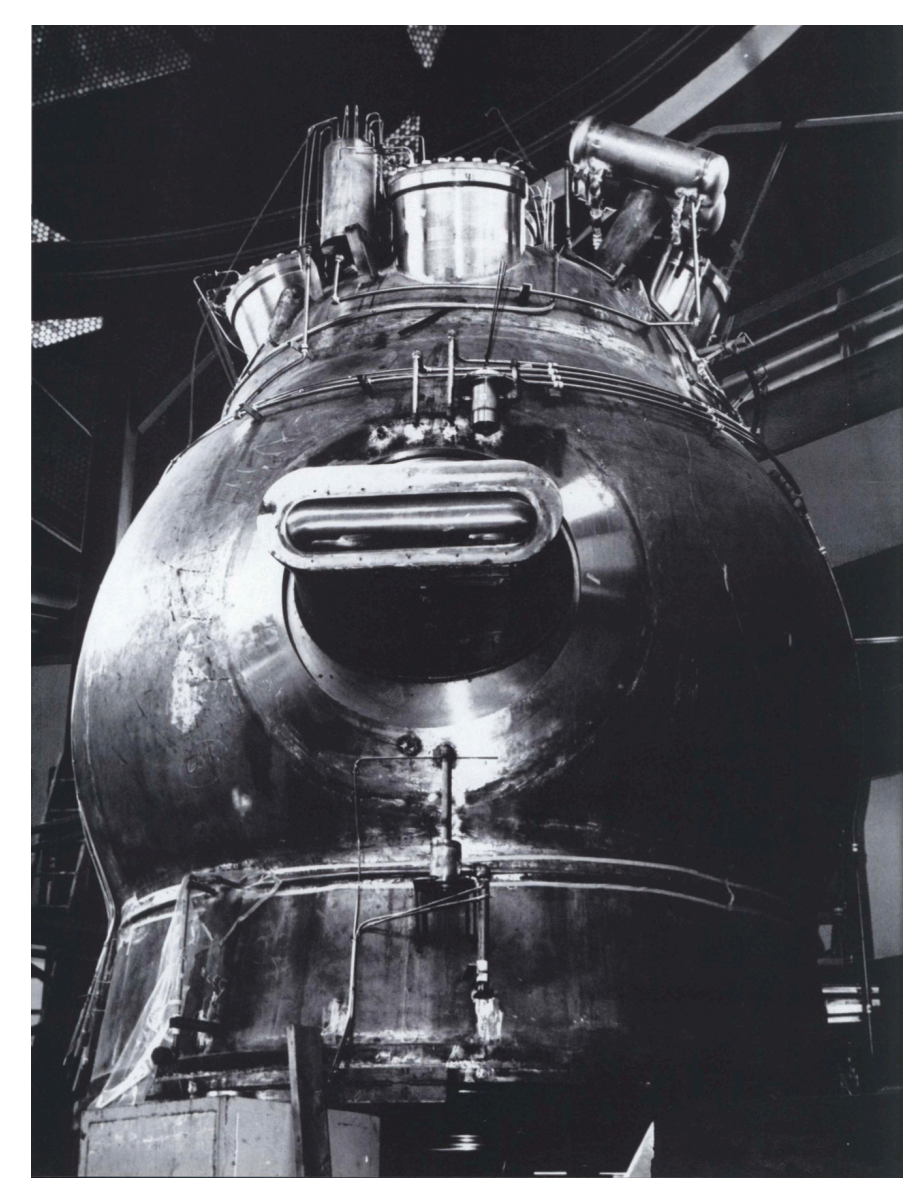

Baltay et al. Reflections on the 15-Foot Bubble Chamber at Fermilab (1988) 


\section{Hadron tensor}

Lattice Calculation of Neutrino-Nucleon Cross Section

Keh-Fei Liu ${ }^{1}$ (liu@g.uky.edu), Terrence Draper ${ }^{1}$, Jian Liang ${ }^{1}$, G. Wang ${ }^{1}$, Yi-Bo Yang ${ }^{2}$, and Yong Zhao

Neutrino nucleus cross-section factorizes into product of lepton and hadron tensors

$$
\begin{aligned}
W_{\mu \nu}\left(q^{2}, \nu\right) & =\int \frac{d^{4} x}{4 \pi} e^{i q \cdot x}\left\langle N\left(p^{\prime}\right)\left|J_{\mu}(x) J_{\nu}(0)\right| N(p)\right\rangle \\
& =\int \frac{d^{4} x}{4 \pi} e^{i q \cdot x} \sum_{f}\left\langle N\left(p^{\prime}\right)\left|J_{\mu}(x)\right| f\right\rangle\left\langle f\left|J_{\nu}(0)\right| N(p)\right\rangle
\end{aligned}
$$

Hadron tensor calculations require inverse Laplace transform of LQCD 4pt functions, exploratory calculations underway using Backus-Gilbert, maximum entropy, Bayesian reconstruction

Below inelastic thresholds, nucleon hadron tensor equal to square of elastic form factors

Above inelastic thresholds, nucleon hadron tensor accesses sum over all intermediate states and could inform nuclear models of resonance, transition, and DIS regions

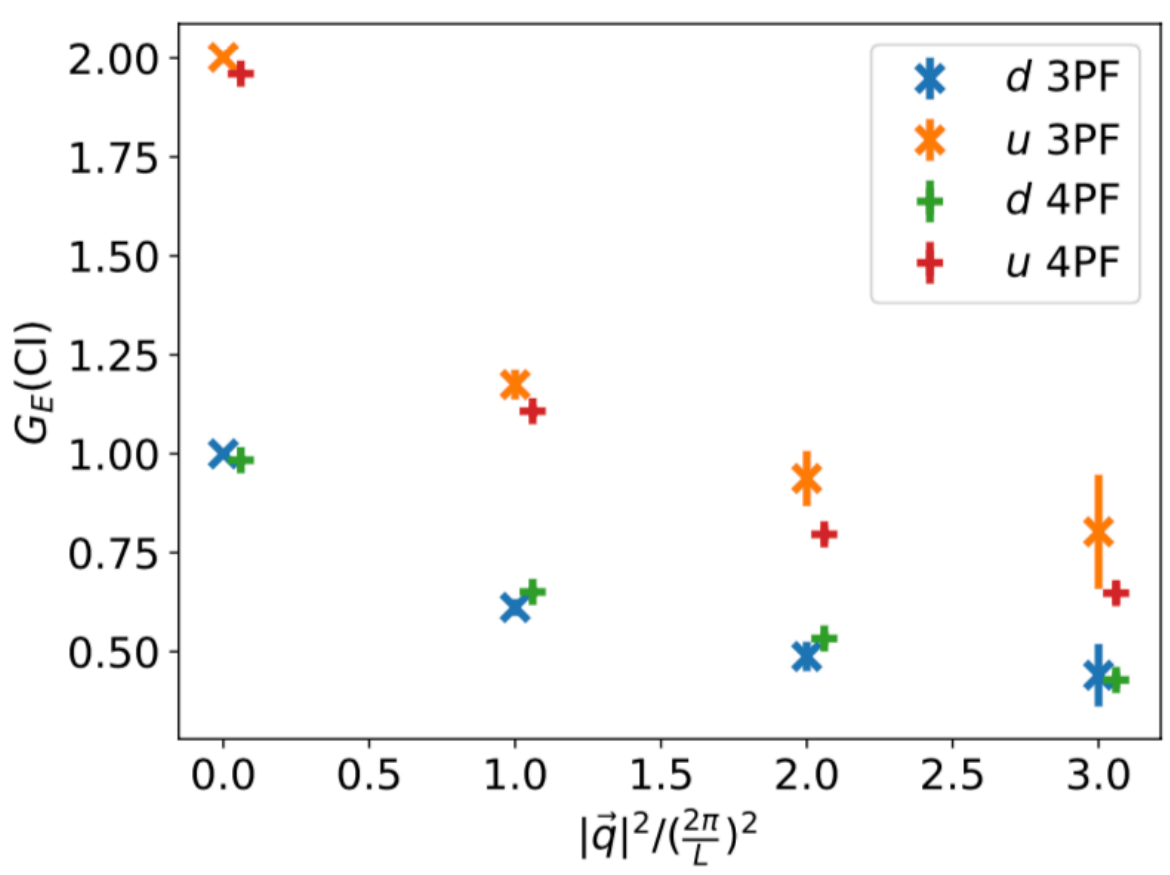




\section{Resonance production}

$\pi$ production essential for reproducing cross-section at energies above quasi-elastic region

Exclusive $\pi$ production amplitudes important for neutrino Monte Carlo event generators

$\Delta$ resonance contributions important and poorly known ingredient to nuclear models of resonance region

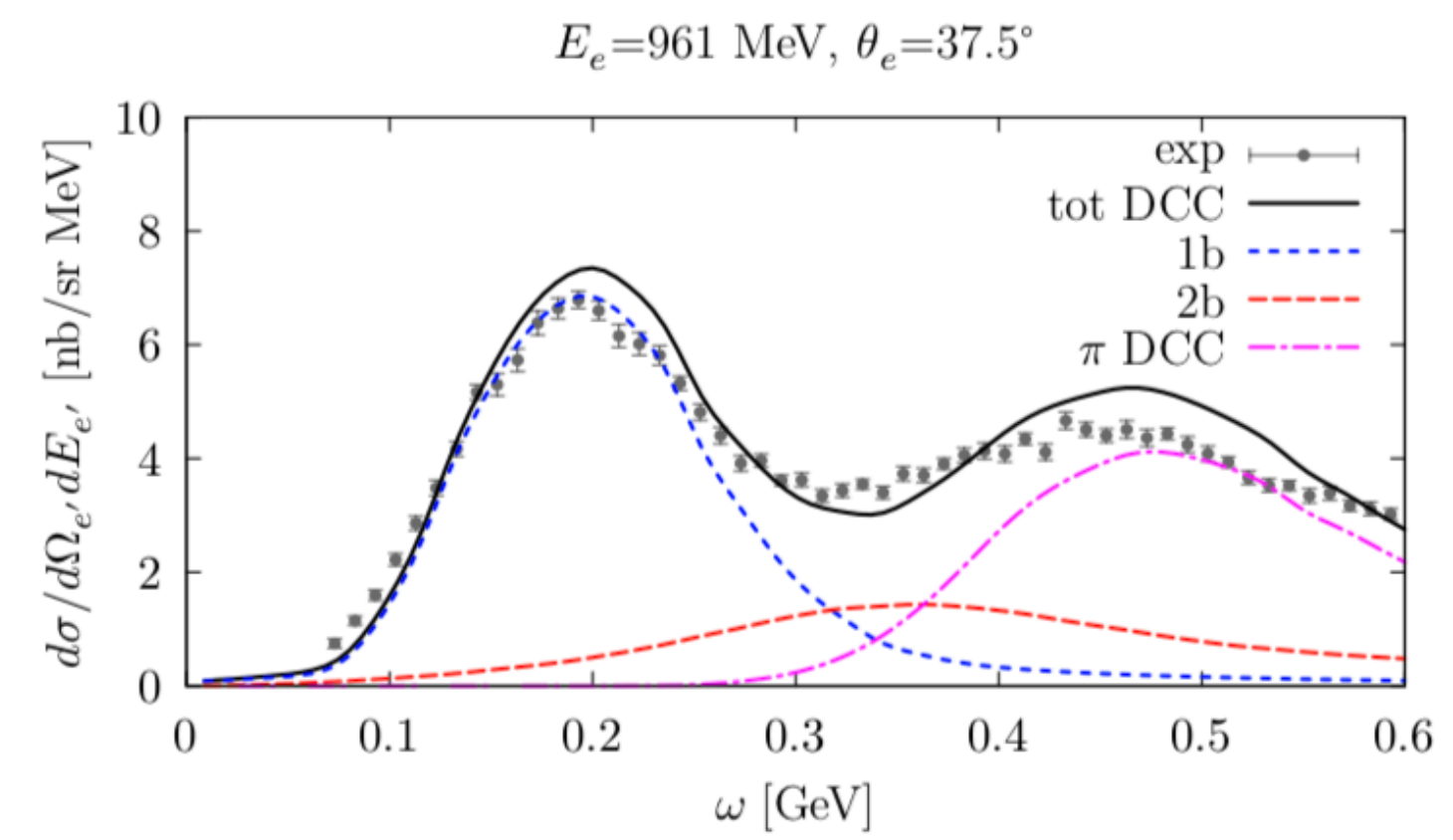

Rocco, Nakamura, Lee, Lovato, PRC 100 (2019)

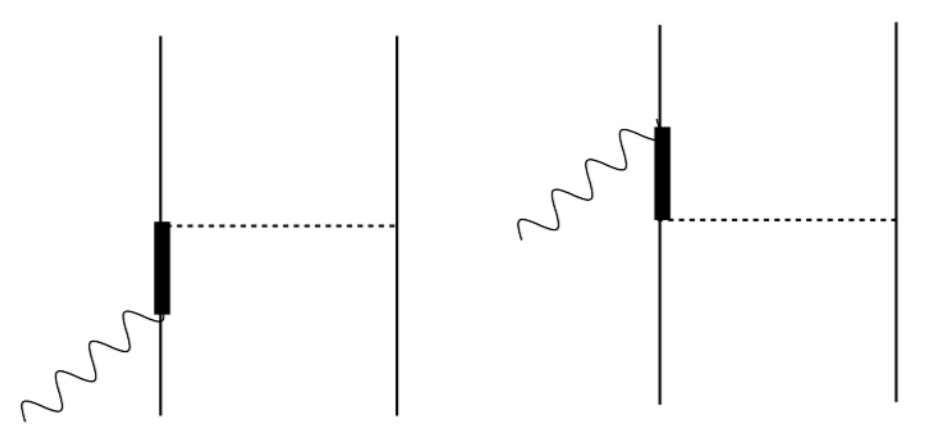

Exploratory LQCD calculations of $N, \pi, \Delta$ performed at heavy quark masses where $\Delta$ stable

Leinweber, Draper, Woloshyn PRD 48 (1993)

$\ldots$

Alexandrou et al, PRD 77 (2008)

Fully controlled LQCD calculations at physical point require sophisticated finite-volume formalism and numerical techniques for $N \pi$ scattering states 


\section{Two-body currents}

$E_{e}=961 \mathrm{MeV}, \theta_{e}=37.5^{\circ}$

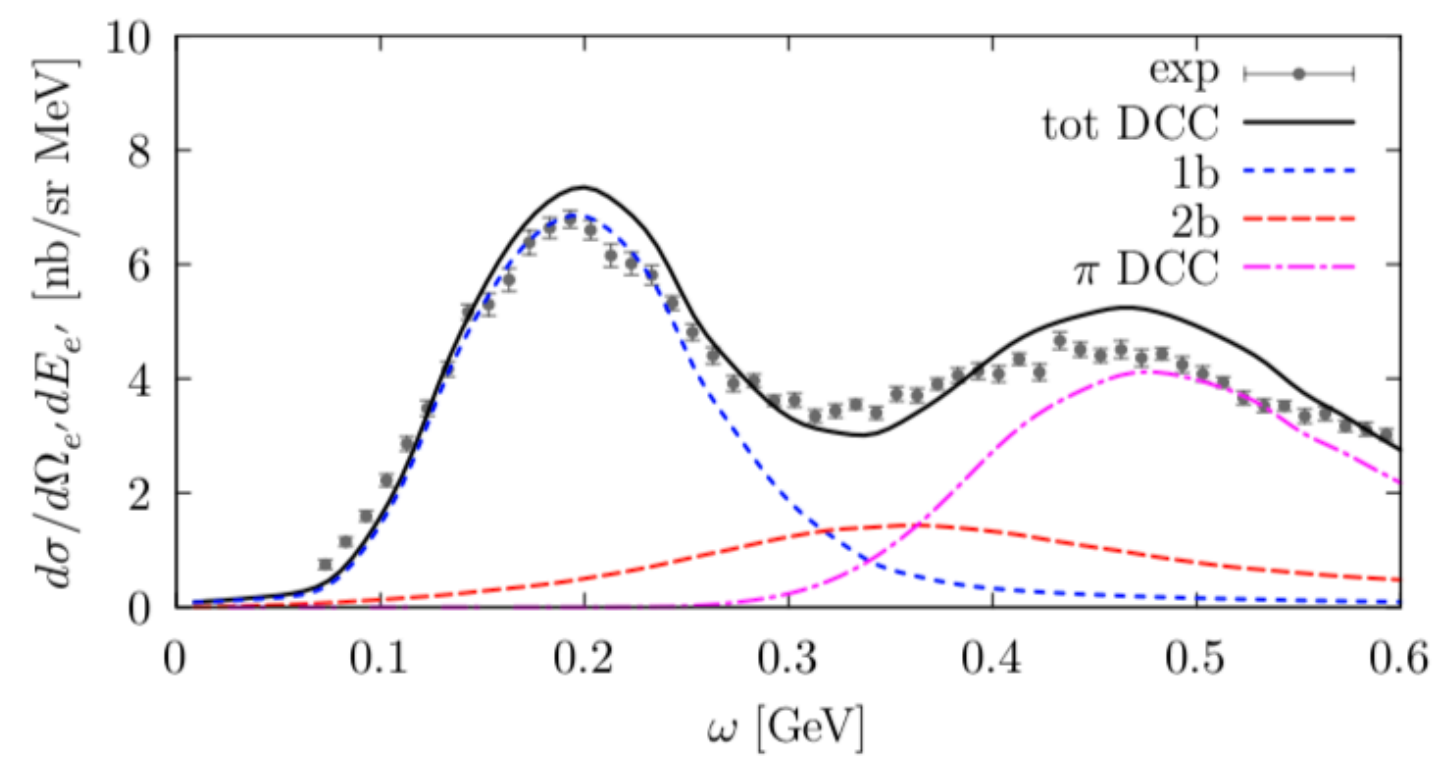

Rocco, Nakamura, Lee, Lovato, PRC 100 (2019)

$A=30-50$ nuclei have $O(30 \%)$ smaller axial couplings than predicted by shell model with single-particle couplings

Multi-nucleon correlations and currents needed to reproduce $\beta$-decay results from experiment
Two-body current effects are essential for reproducing $\nu A$ scattering data in shallow inelastic region

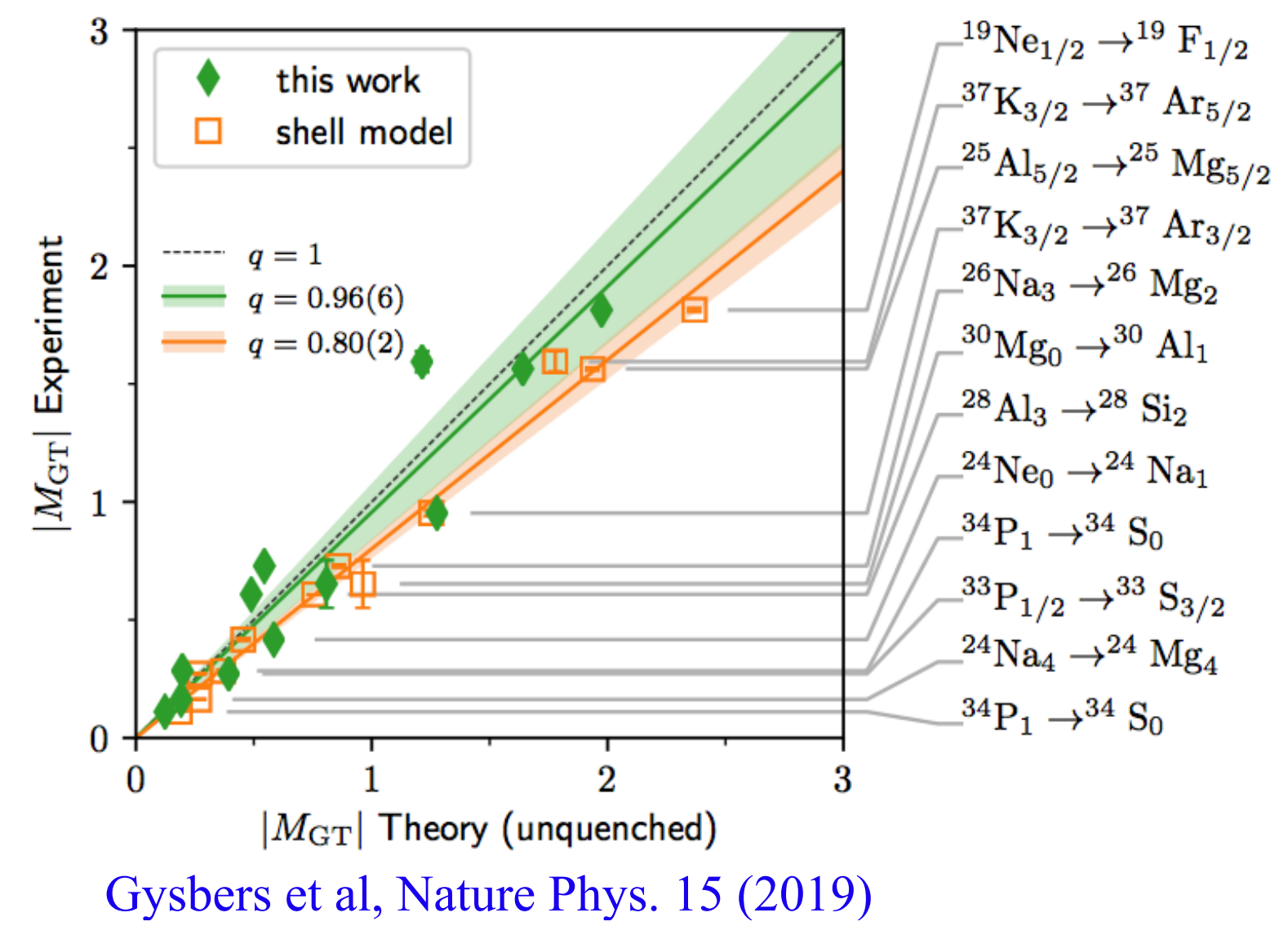




\section{Two-body currents}

Constraints on two-body axial currents obtained by matching LQCD and EFT calculations in a box with a background axial field

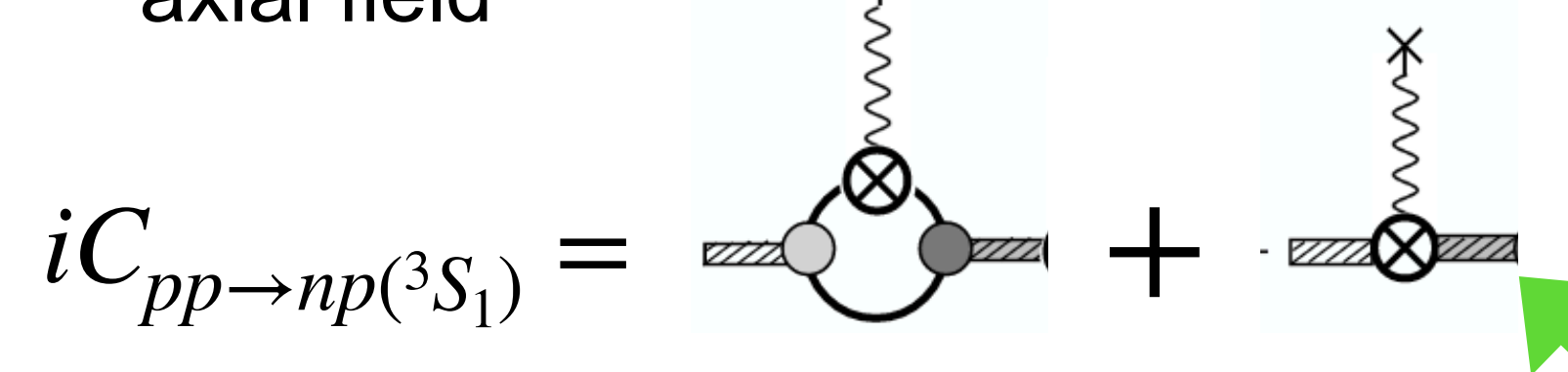

$$
\mathscr{M}_{p p \rightarrow n p\left({ }^{3} S_{1}\right)}=g A(1+S)-L_{1 A}
$$

\section{Short-distance QCD}

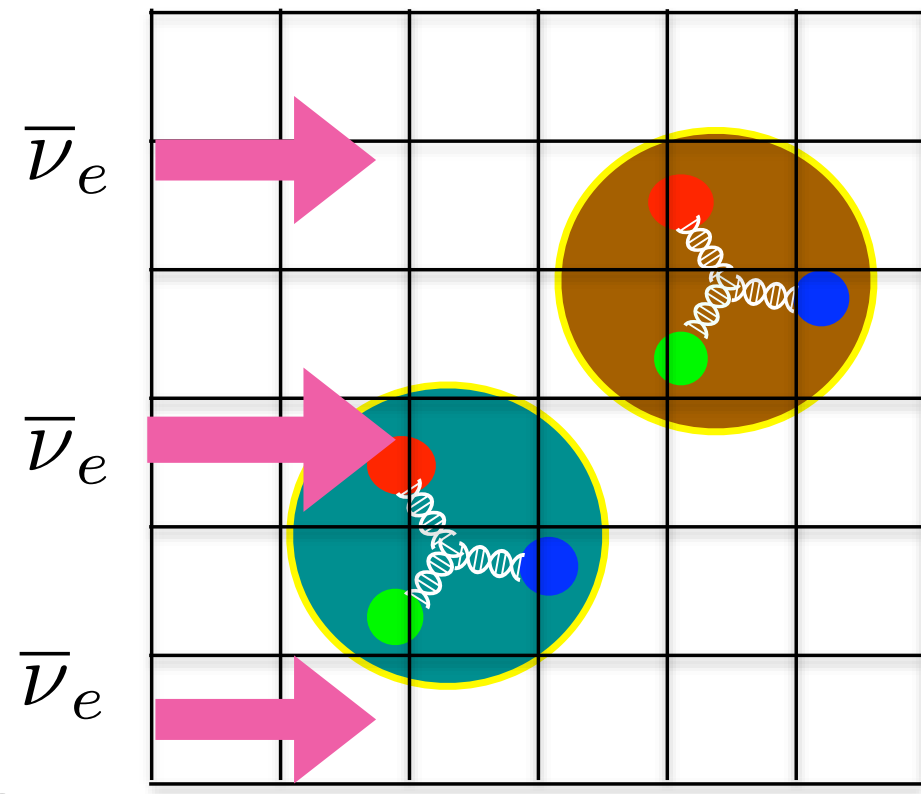

Used for exploratory LQCD determination of $L_{1 A}$ Savage et al [NPLQCD], PRL 119 (2017)

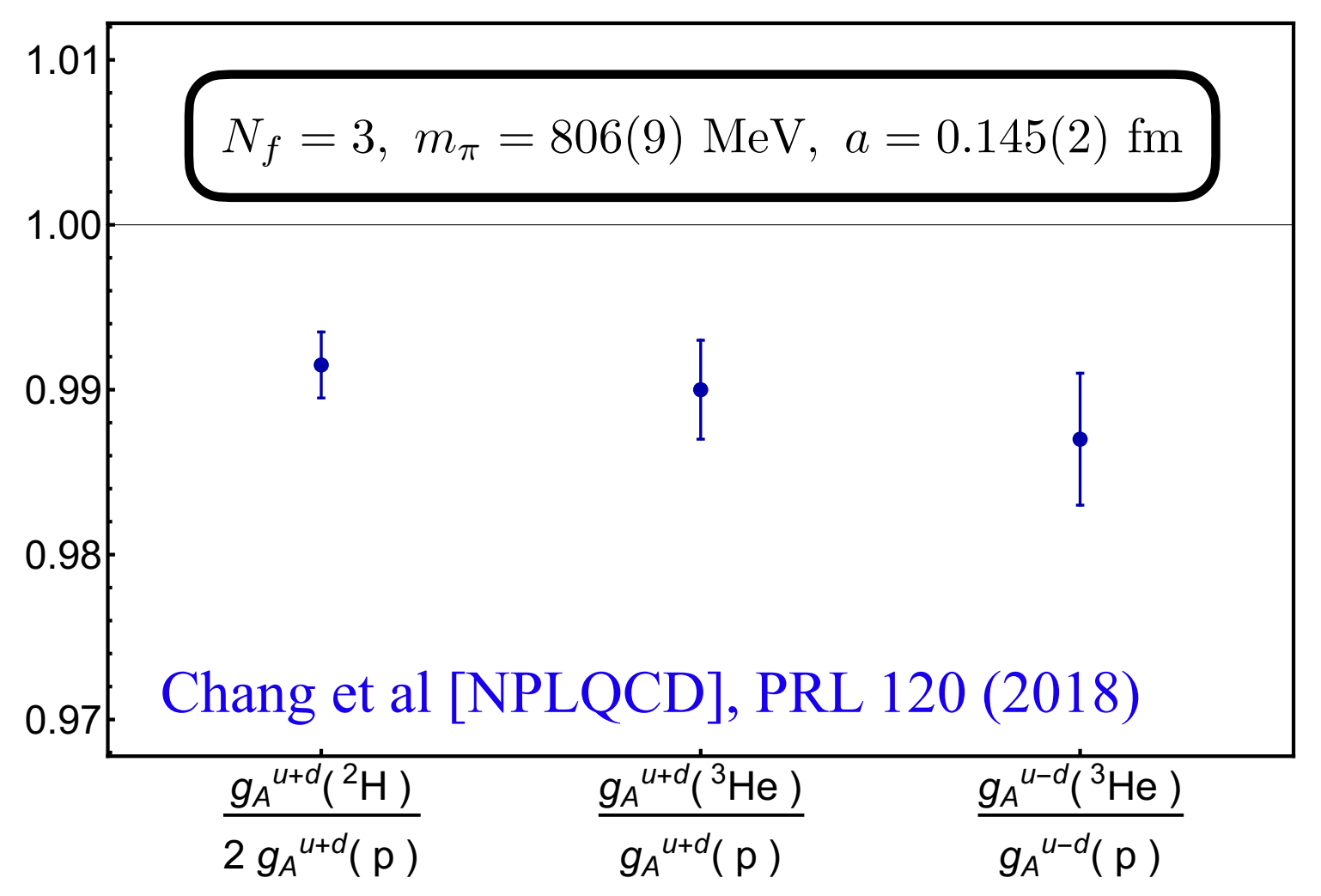

LQCD calculations of $A=2-3$ matrix elements sensitive to two-body currents performed at unphysically heavy quark masses

Further study of $N N$ excited states needed to understand discrepancies between these calculations and recent results using different interpolating operators:

Francis et al, PRD 99 (2019) Hörz et al, arXiv:2009.11825 


\section{Nucleon / nuclear PDFs}

At very high energies, cross-sections factorize into convolutions of perturbative quark-and-gluon-level amplitudes and nonperturbative PDFs

Some aspects of PDFs are experimentally well-known, but others (e.g. flavor dependence, nuclear effects) are not

LQCD constraints can improve global analyses of isovector polarized PDFs
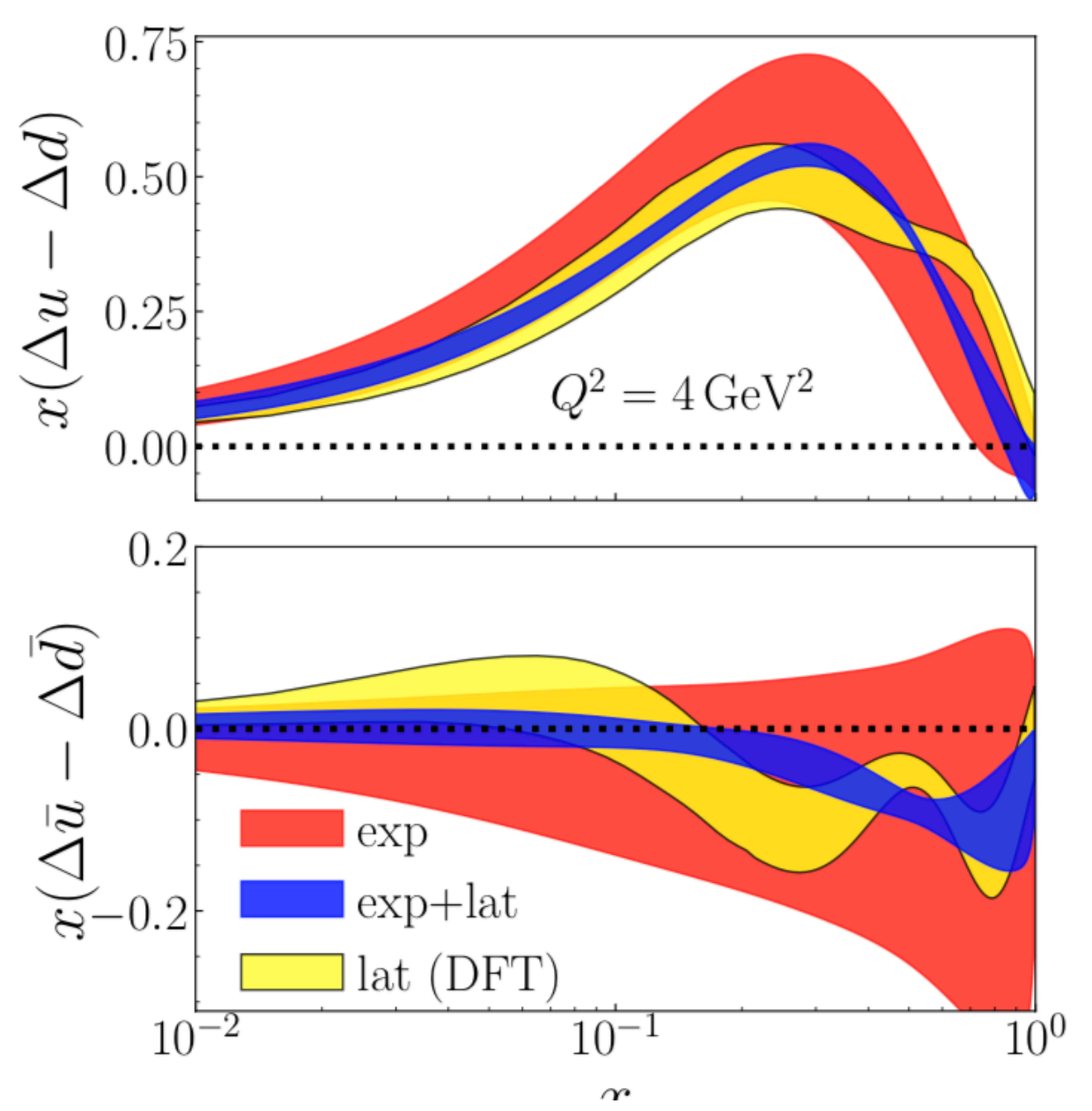

Bringewatt et al [JAM], arXiv:2010.00548
LQCD constraints can also improve global analyses of nuclear PDFs

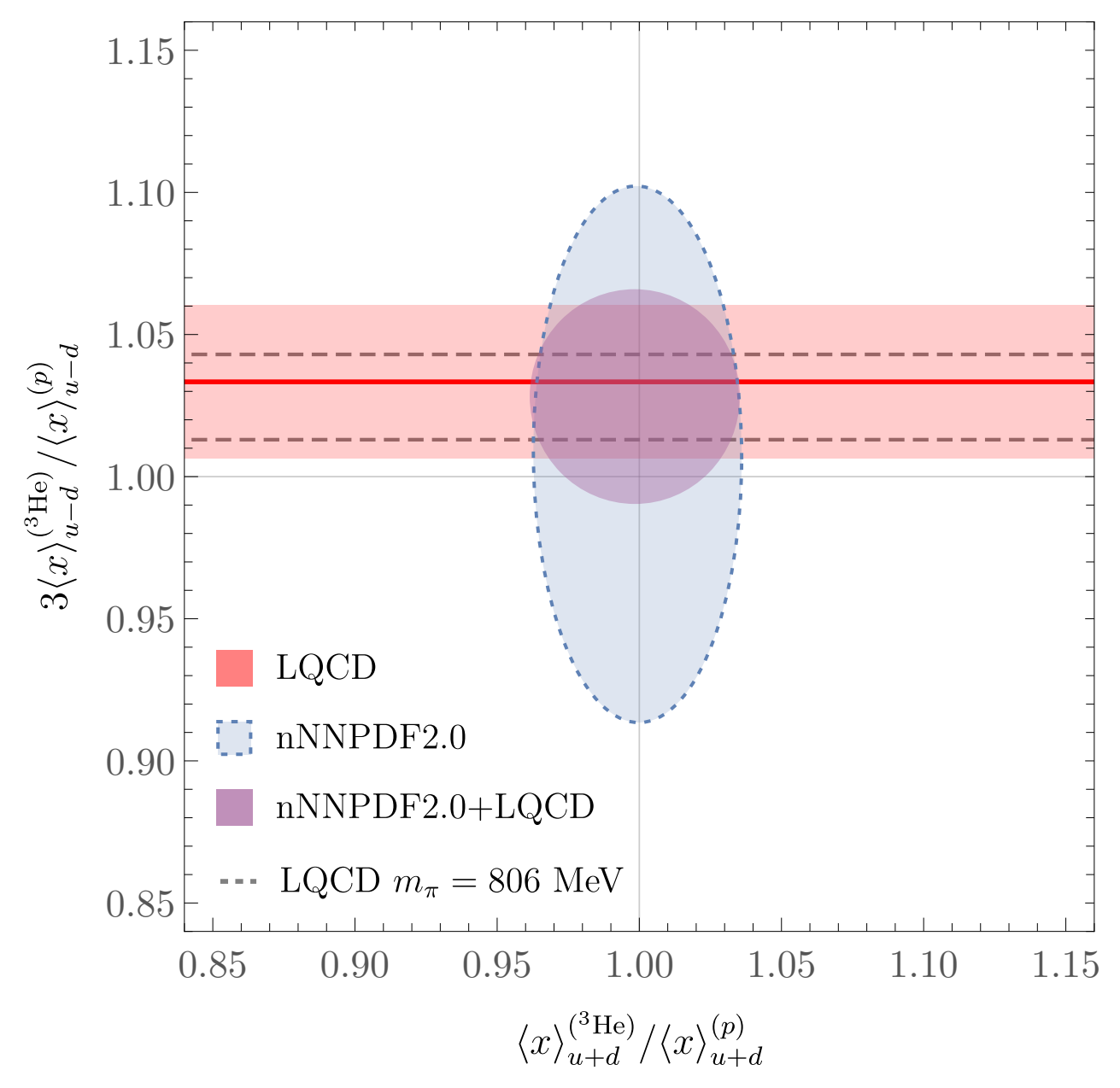

Detmold et al [NPLQCD], arXiv:2009.05522 


\section{Conclusions}

Describing $\nu A$ scattering from the Standard Model requires control of QCD over a wide range of scales and physics processes

Challenging but possible through lattice QCD + nuclear many body methods

Nucleon form factors
Hadron tensor
Resonance production
Two-body currents
PDF constraints
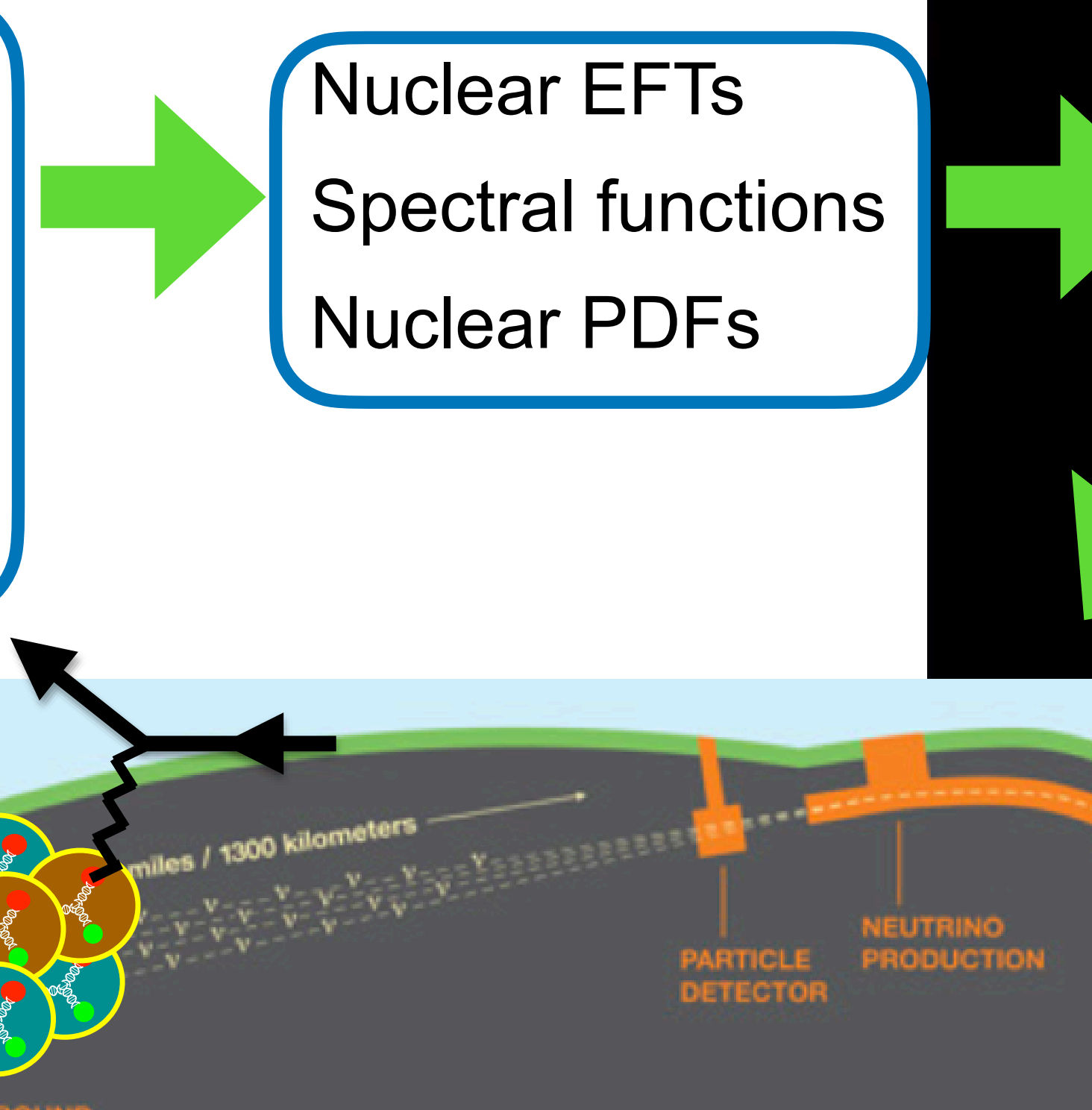